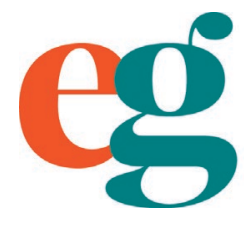

\title{
Violencia sexual en el trabajo de campo: autoetnografía a dos voces
}

\section{Sexual Violence During Fieldwork: Autoethnography in Two Voices}

\author{
Virginia Romero Plana ${ }^{{ }^{*}}$ \\ Luz Martínez Santamaría ${ }^{2}$
}

Recibido: 30 de octubre

\author{
1*Universidad de Sonora, Hermosillo, México. email: virgyromero@gmail.com, \\ Ohttps://orcid.org/0000-0002-9149-0572?lang=es \\ ${ }^{2}$ Investigadora asociada al Museo Nacional de Sicán, Perú. email: \\ luz.martinez.santamaria@gmail.com, (1) https://orcid.org/oooo-ooo1-7279- \\ $483 \mathrm{X}$ \\ *Autora para correspondencia
}

\section{0}

Aceptado: 11 de mayo de 2021

Publicado: 14 de junio de 2021

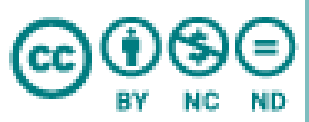

Esta obra está protegida bajo una Licencia Creative Commons Atribución-NoComercialSinDerivadas 4.0 Internacional (CC BY-NC-ND 4.0)

\section{Resumen}

Este artículo analiza, desde la crítica feminista y decolonial, la violencia sexual hacia las mujeres durante la formación universitaria y particularmente en el trabajo de campo, como un aspecto microsocial de la estructura patriarcal $\mathrm{y}$ de las desigualdades sufridas por las mujeres. Desde un enfoque autoetnográfico, a partir del relato de nuestras experiencias de violencia sexual, identificamos cómo opera la violencia de género en el trabajo de campo y rescatamos las respuestas a nuestras denuncias y el impacto en

CÓMO CITAR: Romero, Virginia y Martínez, Luz. (2021). Violencia sexual en el trabajo de campo: autoetnografía a dos voces. Revista Interdisciplinaria de Estudios de Género de El Colegio de México, 7, e717. doi: http://dx.doi.org/10.24201/reg.v7i1.717 
nuestras vidas y trayectorias académicas. Ante la falta de preparación de las universidades para hacer frente a este problema se concluye que es urgente generar un diálogo entre instituciones educativas y voces feministas para reconocer y eliminar la violencia de género.

Palabras clave: violencia sexual; acoso sexual; trabajo de campo; autoetnografía; antropología cultural; crítica feminista.

\begin{abstract}
This article is a feminist and decolonial critical analysis of sexual violence against women studying at university and particularly carrying out field work, as a micro-social aspect of the patriarchal structure and the inequalities suffered by women in our society. Our autoethnographic approach is based on our own experiences of sexual violence; we identify instances of gender violence in field work, and we examine the responses to our complaints and the impact on our lives and academic careers. Considering that universities are unprepared to tackle this problem, our conclusion focuses on the urgent need for a dialogue between educational institutions and feminist voices to recognize and eliminate gender violence.
\end{abstract}

Key words: sexual violence; sexual harassment; fieldwork; autoethnography; cultural anthropology; feminist critique.

Where to from here?

Tarana Burke

Este artículo es una reflexión, desde la crítica feminista y decolonial, acerca de la violencia sexual que vivimos dos antropólogas en el trabajo de campo realizado durante nuestros estudios de posgrado. El objetivo es analizar cómo opera la violencia de género en la academia, en general, y en el trabajo de campo etnográfico, en particular. Para ello, proponemos una autoetnografía a dos voces que, en sintonía con los movimientos sociales de denuncia virtual como \#MeToo o \#Cuéntalo, busca exponer la normalización y ubicuidad de la violencia hacia las mujeres y el enorme impacto que ésta tiene en la vida y trayectoria profesional de quienes la vivimos. Elegimos la autoetnografía como una apuesta para romper 
las bases que sostienen la falsa idea del "etnógrafo sin género" en la producción del conocimiento antropológico, reivindicando la experiencia personal como política y ligada al activismo feminista.

El texto comienza con una revisión teórica acerca de la violencia sexual hacia las mujeres en el trabajo de campo etnográfico y los silencios de las universidades ante las violencias de género. Sigue un apartado metodológico donde se explora la autoetnografía como método para el abordaje de la violencia a partir de la narrativa experiencial y se presenta una propuesta analítica desde la crítica feminista. A continuación, los resultados de esta exploración son analizados en dos ejes: las voces de denuncia y las trayectorias profesionales y vitales. Finalmente, este artículo concluye con algunas propuestas para que las universidades empiecen a enfrentar el problema de la violencia sexual ejercida contra las mujeres.

\section{Violencia sexual, vulnerabilidad en el campo y silencio en la universidad}

El trabajo de campo es el eje central de la investigación antropológica. Es un contexto físico, temporal, simbólico y epistémico que se ha construido —y romantizado - a partir de los relatos de hombres en el contexto del colonialismo e imperialismo europeo y norteamericano. En la actualidad existe una noción institucionalizada del trabajo de campo como un rito de paso masculinizado o como un ejercicio de resistencia individual (Berry, Chávez, Cordis, Ihmoud y Velásquez, 2017). Para el ámbito latinoamericano, Espitia, Ojeda y Rivera (2019) advierten cómo la enseñanza de las técnicas etnográficas y del trabajo de campo sigue el arquetipo del etnógrafo masculino y androcéntrico, que fomenta, oculta y justifica distintas formas de violencia contra los cuerpos que no se ajustan al perfil. La idea del etnógrafo sin género es una cobertura ideológica que perpetúa la desigualdad y cuya consecuencia es la invisibilización de la violencia sexual que vivimos las mujeres antropólogas. Este planteamiento mantiene el carácter supuestamente objetivo que borra las cuestiones de raza,

género y clase en el proceso de investigación y afirma una postura neutral que niega las formas coloniales y extractivistas de producción de conocimiento. 
El planteamiento de que no existe el género en el trabajo de campo ha sido ampliamente cuestionado por antropólogas feministas desde la década de 1970 (Berry et al, 2017; Escobar, 2018; Kloß, 2017). Una de las pioneras en hablar sobre la violencia sexual que vivió en Etiopía fue Gunilla Bjerén, bajo el pseudónimo de Eva Moreno, quien afirma que es imposible mantener la ficción del "yo sin género" en el trabajo de campo, donde "los antropólogos no son acosados o violados, las mujeres sí" (Moreno, 2005, p. 246). A pesar de esto, la violencia sexual continúa siendo un tema marginal -0 inexistente - en las discusiones metodológicas de la formación antropológica en muchas universidades.

La actitud de la academia frente a la violencia de género ha sido de negación y silencio, culpabilizando a las investigadoras individualmente. El primer y paradigmático caso fue el de la antropóloga norteamericana Henrietta Schmerler, una estudiante de Ruth Benedict en la Universidad de Columbia a principios del siglo XX, quien fue violada y asesinada cuando hacía trabajo de campo en una comunidad Apache de Arizona en 1931. De su violación y muerte fue acusado un nativo, pero tanto la universidad como la prensa de la época culparon a Henrietta por lo sucedido (Steffen, 2017). Su caso ha seguido apareciendo en los medios y debates antropológicos hasta hace poco con el mismo sesgo de culpabilización, argumentando una supuesta falta de conocimiento de Henrietta de las costumbres de la comunidad (Schmerler y Steffen, 2018).

Casi un siglo después prevalece la táctica de silenciar y culpar a las investigadoras en un intento fallido por justificar la estructura patriarcal de la academia y de la sociedad en general. A pesar de que la antropología tiene las herramientas para construir la comprensión de este fenómeno, todavía es renuente a hacerlo, ya que confrontar este tipo de violencia en la disciplina nos obliga a repensar los métodos, los marcos epistemológicos y nuestras formas de producción de conocimiento, además de aceptar su imbricación en dinámicas de poder y violencia (Huang, Lu, Macdougall y Steffen, 2018).

La resistencia a enfrentar esta violencia en la academia española y latinoamericana, al igual que su activo y continuo silenciamiento, tiene su razón en conservar la "reputación" de los agresores y de las instituciones que los cobijan y encumbran. Estas últimas no castigan la 
violencia hacia las mujeres, sino que las culpa y revictimiza, a pesar de la creación y reciente implementación de leyes y políticas.

El victimario necesita de toda una estructura de complicidad que permita que suceda el abuso, además de una cultura de retaliación y de gaslighting a quienes se atreven a denunciar, lo que socava la posibilidad de obtener justicia.

Las consecuencias del silenciamiento y bloqueo hacia el conocimiento de estas situaciones son enormes para las mujeres que las sufren. La violencia de género en el trabajo de campo conlleva retrasos, desviación hacia otras áreas o, incluso, el abandono de la carrera académica (Nelson, Rutherford, Hinde y Clancy, 2017). En un comentario a su artículo Rape in the Field (2005), Bjerén reflexiona sobre los efectos de la violación que sufrió en Etiopía durante la investigación y destaca cómo tuvo que distanciarse del material que había recopilado en el campo, lo que provocó un cambio en el rumbo de su carrera. Otra consecuencia fue aceptar que ser mujer significaba, dentro y fuera de la academia, enfrentar las injustas diferencias entre los sexos (Bjerén, 2017). En numerosos relatos sobre violencia de género, mujeres antropólogas han señalado el extraordinario trabajo afectivo, físico e intelectual que requiere reafirmar nuestra legitimidad como investigadoras (Berry et al, 2017).

En lugar de silenciar estas experiencias es necesario incluirlas en el análisis de los datos adquiridos y reflexionar sobre ellas en la escritura etnográfica (Kloß, 2017). Las antropólogas que develamos públicamente episodios de violencia sexual lo hacemos sabiendo que esto puede poner en riesgo nuestras carreras, que se va a dudar de nuestra profesionalidad y que nos exponemos todavía a más daños emocionales (Huang et al, 2018).

\section{Antropología después del \#MeToo: romper el silencio para la transformación social}

En octubre de 2017, después de las acusaciones de varias actrices contra el productor Harvey Weinstein, la actriz Alissa Milano llamó a tuitear \#MeToo. En las primeras 24 horas el hashtag \#MeToo obtuvo 12 millones de tuits, lo que mostró la magnitud del problema de la 
violencia sexual hacia las mujeres y el fracaso en escuchar y tomar en serio a las sobrevivientes cuando revelan sus experiencias (Fileborn y Lonely-Howes, 2019). Posteriormente, surgen otros movimientos similares en contextos locales, como \#Cuéntalo para el ámbito hispanohablante, y plataformas de ayuda y asesoramiento a mujeres que sufren violencia sexual, como Time's Up, u otras propias de la antropología y la academia, como \#MeTooAnthro o \#MeTooPhD, que buscan visibilizar la violencia sexual hacia las jóvenes investigadoras.

Este movimiento ha logrado evidenciar la ubicuidad de la violencia sexual hacia las mujeres en todo el mundo y en todos los ámbitos, exponiéndola al debate público. Por medio del hashtag \#MeToo se denuncian situaciones de violencia muy distintas, desde "piropos" o insinuaciones hasta violaciones, que convergen en los mismos mecanismos de abuso, intimidación y fuerza, basados en la única intencionalidad de controlar a las mujeres, negándoles la autonomía de sus propios cuerpos. La académica feminista Liz Kelly (1988) definió esto como un continuum de violencia sexual.

Una debilidad del movimiento es la minimización del papel de la etnia, las condiciones socioeconómicas y las personales, que se diluyen en la comunalidad de la violencia. Indicativo de esta situación es el hashtag de \#MeToo que fue creado por una afroamericana, Tarana Burke en el año 2006, pero convertido en lo que es ahora por el uso hecho por mujeres blancas ${ }^{1}$. El mensaje de Burke es romper el silencio, ya que éste es una poderosa herramienta en la perpetuación de la violencia hacia las mujeres afrodescendientes.

Una de las principales críticas al \#MeToo es la facilidad con que discursos esencialistas sobre la violencia sexual pueden reforzar ideas y políticas conservadoras sobre la sexualidad y convertirse en una "caza de brujas", a partir de la idea de que exponer y avergonzar a los hombres públicamente puede ser fácilmente desviado con fines políticos o personales (Salter, 2018). Los “escándalos" de personajes masculinos destacados en sus disciplinas ${ }^{2}$ son una

\footnotetext{
${ }^{1}$ Las autoras del artículo entendemos que podemos hablar sobres nuestras experiencias de violencia sexual por nuestra posición como mujeres con privilegios, pero queremos unirnos a esta ruptura del silencio.

${ }^{2}$ En el campo de la antropología, el caso más paradigmático ha sido el de la revista HAU (Journal of Ethnographic Theory), que generó el denominado \#HAUTalk donde se discutían los abusos de poder del entonces director de la revista a jóvenes investigadoras e investigadores, así como el encubrimiento e inacción
} 
muestra más de la normalización de la violencia hacia las mujeres (Segato, 2010). En este sentido, Burke apunta que nos debemos mover de los titulares "escandalosos" e individuos específicos hacia las causas sistémicas de la violencia sexual.

El movimiento \#MeToo se basa en el reconocimiento de experiencias de violencia sexual que se comparten públicamente en redes sociales. Se ha resaltado que esta forma de expresión digital puede apuntar a un ejercicio excesivamente individualista, sin embargo, ha permitido la creación de espacios de encuentro entre mujeres y otro tipo de activismo (asociaciones, protestas, marchas y diversas vías de exposición de denuncia). Ha creado comunidad, tanto para transformaciones personales hacia la superación del trauma de las sobrevivientes, como para la búsqueda de significados de cambio en las condiciones que hacen posible la prevalencia de la violencia sexual (Mendes, Ringrose y Keller, 2018).

Es importante resaltar que, aunque su formato virtual y global lo sean, el \#MeToo no es una novedad en la lucha feminista y debe enmarcarse en los movimientos antiviolencia sexual que surgieron en la década de 1970 con grupos de mujeres que se reunían para contar sus vivencias (Fileborn y Lonely-Howes, 2019). Estos colectivos inspiraron el pensamiento y la crítica del momento donde la experiencia se entendió como construida y definida social y colectivamente en la lucha política (Mulinari y Sandell, 1999). Esta primera teorización de la experiencia fue criticada posteriormente como "universalizadora" de la experiencia femenina. De Lauretis (1986) argumenta que el feminismo debe alejarse de una construcción hipotética de la mujer (incluyendo la clase, la raza y la sexualidad) aunque reivindica la experiencia como política y como una forma para repensar nuestra cultura.

En el encuentro con el posmodernismo, Joan Scott (1991) pone en duda la experiencia como punto de partida para teorizar la construcción de discursos de feminidad, ya que esencializa la identidad y cosifica al sujeto. Insiste en el carácter discursivo de la experiencia y en la política de su construcción: "La experiencia es a la vez siempre una interpretación y algo que

por parte de los otros responsables de la publicación. Mientras escribíamos este artículo, el periódico estudiantil de la universidad de Harvard, The Crimson, destapó el caso de tres antropólogos señalados por acoso y abuso sexual y protegidos durante décadas en sus puestos. Entre los académicos señalados está un reconocido andinista, acusado de abusos en las misiones de campo arqueológicas en el norte de Perú. 
necesita ser interpretado. Lo que cuenta como experiencia no es evidente ni sencillo; siempre es controvertido y, por lo tanto, siempre político" ${ }^{3}$ (Scott, 1991, p. 797). Subraya que no son las personas las que tienen experiencias, sino que las personas son construidas por medio de éstas. En este tenor, el uso de la autoetnografía busca no poner la experiencia como el origen, sino como parte de lo que construye nuestras subjetividades como mujeres, académicas y feministas. Como de Lauretis y Scott, queremos reivindicar la potencialidad de la experiencia como política para entender, visibilizar y transformar una realidad que afecta a muchas mujeres.

\section{Aspectos metodológicos}

Este trabajo es una reflexión profunda a dos voces que explora, construye sentido e interpreta la violencia sexual desde un método introspectivo vivencial: la autoetnografía, que resalta la concepción epistemológica de la realidad desde su relación con un contexto, una serie de creencias y ciertos modos de pensar (Camacho y Marcano, 2003). Elegimos la autoetnografía como metodología para ubicar en el centro nuestra experiencia, no sólo para entender cómo opera la violencia de género en el trabajo académico, sino también como una herramienta para cambiar esa realidad que contribuye al borrado de las mujeres en la práctica etnográfica.

\section{Autoetnografía feminista}

La autoetnografía se fundamenta en una reflexión teórico-crítica de análisis profundo sobre determinados aspectos de experiencias, convivencia social, emociones y acciones (Gregorio, 2014). En esta versión de la etnografía la persona investigadora se destaca como eje clave del análisis de la realidad social, comprendida ésta en un límite espaciotemporal y sociocultural. Se convierte, por lo tanto, en una indagación de la relación existente entre la vivencia de quien investiga y el espacio cultural donde se encuentra. La experiencia

3 "Experience is at once always already an interpretation and something that needs to be interpreted. What counts as experience is neither self-evident nor straightforward; it is always contested, and always therefore political". 
individual se aborda desde una mirada directa y sentida, que pertenece a una relación construida entre las personas y un entramado cultural específico.

Esta autoetnografía nos posiciona en dos frentes como sujetas-investigadoras: se construye una relación directa (indiscutiblemente subjetiva) a partir del relato de nuestras vivencias como protagonistas, pero también una relación indirecta con la cultura, conformada desde lineamientos teóricos sobre el fenómeno. La autoetnografía dispone de "la propia experiencia como una forma de llegar a la dimensión cultural, pero también a la política [...] de los fenómenos estudiados, yendo y viniendo de lo local a lo global, de lo individual a lo colectivo" (Esteban, 2004, p. 18).

El potencial de la autoetnografía cada vez se afianza más por reconocidos trabajos que posicionan la conciencia, e incluso la acción política, como meta de los aportes sociales desde la academia (Bénard, 2019; Tilley-Lubbs, 2015; Ellis, 2009). Sin embargo, no somos ajenas a la crítica vertida hacia la autoetnografía y consideramos las desventajas que conlleva realizar un estudio desde el "yo vivencial"; esto implica un distanciamiento con la situación de "las otras y los otros" respecto al problema, centrándonos únicamente en la relación entre nosotras y la cultura. A pesar de que la autorreflexividad ha sido tildada de inconveniente por la subjetividad que conlleva (Finlay, 2002), es ese mismo elemento el que consideramos de valor para un análisis diferente.

Ahondando en la propuesta de Blanco (2012) de sopesar las posibilidades de praxis que presenta una gama amplia de epistemologías y metodologías no tradicionales y siguiendo a Lonergan (2001), consideramos que "el método no es un conjunto de reglas [...]; es más bien un marco destinado a favorecer la creatividad y la colaboración" (p. 9). Por tanto, apostamos por abandonar la metodología convencional y nos arriesgamos a elaborar una autoetnografía a dos voces sobre la violencia sexual en el trabajo de campo, cediendo la palabra a quienes se les había negado como marginales (Grant, 2010) y recuperando las experiencias con una mirada inclusiva (Dubé, 2017). 


\section{Narrativa experiencial}

La narrativa experiencial como una de las técnicas literarias que la autoetnografía acoge en su abanico de posibilidades metodológicas (Blanco, 2012b), no sólo provee del espacio para ir construyendo la vivencia, sino que la ordena y la sistematiza para el posterior análisis desde un esquema subjetivo y original, a partir de un cúmulo de elementos que brotan desde el mismo pensar-sentir-escribir en el ejercicio de introspección de las autoetnógrafas.

Las y los investigadores narran selectivamente epifanías (fenómenos íntimos considerados transformadores), "que surgen y que son posibles porque ellos mismos son parte de una cultura y tienen una identidad cultural particular" (Ellis, Adams y Bochner, 2015, p. 253). La expresión lingüística escrita representa, de alguna manera, el elemento propio de nuestro significado complejo y completo de las experiencias vividas hace casi una década (Lonergan, 2001).

Algunas de las desventajas de la narrativa experiencial han sido el destacado énfasis en la narración (frente a la sistematización pormenorizada) y la semiconfidencialidad de los sujetos (Guerrero, 2014), ya que en el relato aparecen otros actores sociales involucrados en los tejidos socioculturales. Es permanente el dilema ético sobre "cómo es posible no implicar o incluir a otros en [...] el tejido de la trama de nuestra historia" (Douglas y Carless, 2013, p. 98). Sin embargo, consideramos que las experiencias narradas en un relato son las "que procuran modelos de comprensión de la acción colectiva e individual" (Delory-Momberger y Betancourt, 2015, p. 63), ya que no podemos separar las vivencias de los contextos y de las personas que nos rodean y con las que co-construimos las realidades.

Ligado a esto último, otro riesgo de la narrativa vivencial es "el desborde de la intimidad" cuando se tratan cuestiones traumáticas (Arfuch, 2012), ya que los trabajos autoetnográficos abordan "temas delicados, temas tabú, los aspectos sagrados u ocultos de nuestras vidas, y en historias y cuerpos que han sido silenciados, marcados, estigmatizados y olvidados"

(Douglas y Carless, 2013, p. 98). En este tenor, ambas autoras apelamos a nuestro compromiso con las mujeres y con nosotras mismas. 


\section{Ruta metodológica}

Por la delicadeza del tema, la ruta metodológica autoconstruida por las autoras tuvo unos tiempos y unas características peculiares.

\section{Etapa 1: primer con-tacto}

Nuestra relación ${ }^{4}$ se remonta al año 2011 cuando un amigo peruano en común nos puso en contacto a través de una red social, porque él presenció algunas de las violencias que sufrimos ambas en nuestros trabajos de campo por parte de un antropólogo (referido a partir de ahora como J), con quien nuestro amigo trabajaba en aquel momento. $\mathrm{J}$ iba a ayudarnos a entrar en contacto con una comunidad originaria al norte de Perú. En ese momento no se consolidó un vínculo entre nosotras.

\section{Etapa 2: construcción de relatos-espejo}

Tras diez años de distancia, y tres intentos de "mirarnos y reconocernos" (por comunicaciones esporádicas en redes sociales), en 2020 nos unimos como equipo de trabajo, viéndonos a nosotras mismas “como el fenómeno” a estudiar (Ellis, Adams y Bochner, 2015).

El primer ejercicio que desarrollamos fue plasmar, a modo de relato, nuestra experiencia. La memoria es selectiva (Guerrero, 2014); en este sentido, las autoras rescatamos (para leer antes de escribir nuestro relato) textos (diarios y mails) que cada una redactó tras las vivencias. Parte de nuestras narraciones se incorporan en los resultados con algunas ediciones para eliminar lugares, instituciones y nombres personales.

La construcción de un relato individual nos llevó meses. Respetamos el espacio de cada una para armar el escrito de la vivencia. La formación de relatos o el proceso de escritura puede tener distintos tintes; en nuestro caso el relato fue libre sin límite de extensión ni directriz alguna y se acogió al tipo de "escritura confesional-emotiva" (Chang, 2008). En este sentido, una narración en primera persona fue la estrategia para plasmar la subjetividad de la experiencia desde dos "yo vivenciales" multisituados.

\footnotetext{
${ }^{4}$ Las dos investigadoras estudiamos nuestro posgrado en una reconocida universidad madrileña.
} 
Con el relato no sólo buscábamos ofrecerle a la compañera nuestra versión, sino también visualizarla desde una perspectiva más propositiva con nuevos significados y reformulando un sentido de vida (Roscoe y Madoc, 2009). Asimismo, tomamos nuestra escritura reflexiva de la historia como un reflejo de otras connotaciones, que transmite el hecho en sí, y de la identidad femenina que nos define actualmente.

Al igual que Bjerén (2017), quien apunta cómo la escritura de Rape in the Field (1995) le ayudó a deshacerse de "la obligación de recordar" el suceso traumático que vivió, una vez compartido, confirmado por otras personas, documentado y guardado, nosotras también apostamos a que nos ayudaría a sanar y a olvidar, desactivando el dolor del recuerdo.

\section{Etapa 3: lectura "cerca-lejos" de dos historias de violencia}

El siguiente paso fue compartir nuestros relatos para conocer de cerca lo ocurrido desde los ojos de "la otra", quien pronto se convirtió en la persona que construiría una reflexión analítica desde el distanciamiento del relato externo y la conexión del relato propio (un análisis cerca-lejos). Ellis, Adams y Bochner lo denominan "narrativa co-construida" (2015), involucrando varias narraciones sobre un mismo fenómeno, escritas al mismo tiempo, y posteriormente compartidas como la clave para reaccionar ante lo que se pretende analizar. La incorporación de dos relatos rompe, en cierta medida, el carácter cerrado de una autoetnografía, ya que no se abordará sólo la experiencia de una misma, sino dos historias bajo un mismo contexto cultural.

Entender los hechos y reacciones de una realidad en la que hemos estado inmersas implica apropiarnos de la experiencia para repensarla bajo distintos patrones de lógica y conocimiento desde el "yo analista"; y esto sólo puede realizarse bajo la introspección, es decir, volver a pensarnos a partir de la objetivación de ideas y teorías (Lonergan, 2001).

Las autoras delimitamos nuestro rol en este "juego de subjetividad-objetividad" y tras leernos, encontrarnos, separarnos, reconocernos e interpretarnos (a nosotras mismas y a la otra), develamos los matices culturales que construyeron la reflexión crítica de la violencia sexual. 
El lente analítico de los dos relatos se tejió a partir de tres ejes: el contexto sociocultural y económico-político (la sociedad), las relaciones primarias y secundarias y lo que se entiende por comunidad (familia, grupos sociales, escuela...) y la persona en el centro de toda reflexión y resiliencia desde la apropiación de las vivencias.

A partir de este trabajo establecimos tres categorías (Cuadro 1): a) posicionamientos y voces, b) respuestas de las y los actores sociales y c) trayectorias vitales y académicas. Con base en éstas se desprendieron los siguientes aspectos de análisis, los cuales se fueron conformando a lo largo de las conversaciones virtuales de las investigadoras: significados, discursos, trayectorias, expectativas, sororidad, resiliencia y resignificación de la violencia de género. La caracterización de las dos voces reflexivas tiene los siguientes aspectos en común: el hecho de ser mujeres y antropólogas, posicionarnos desde el feminismo y haber experimentado episodios de violencia de género.

\section{Cuadro 1. Propuesta de modelo de análisis para una autoetnografía}

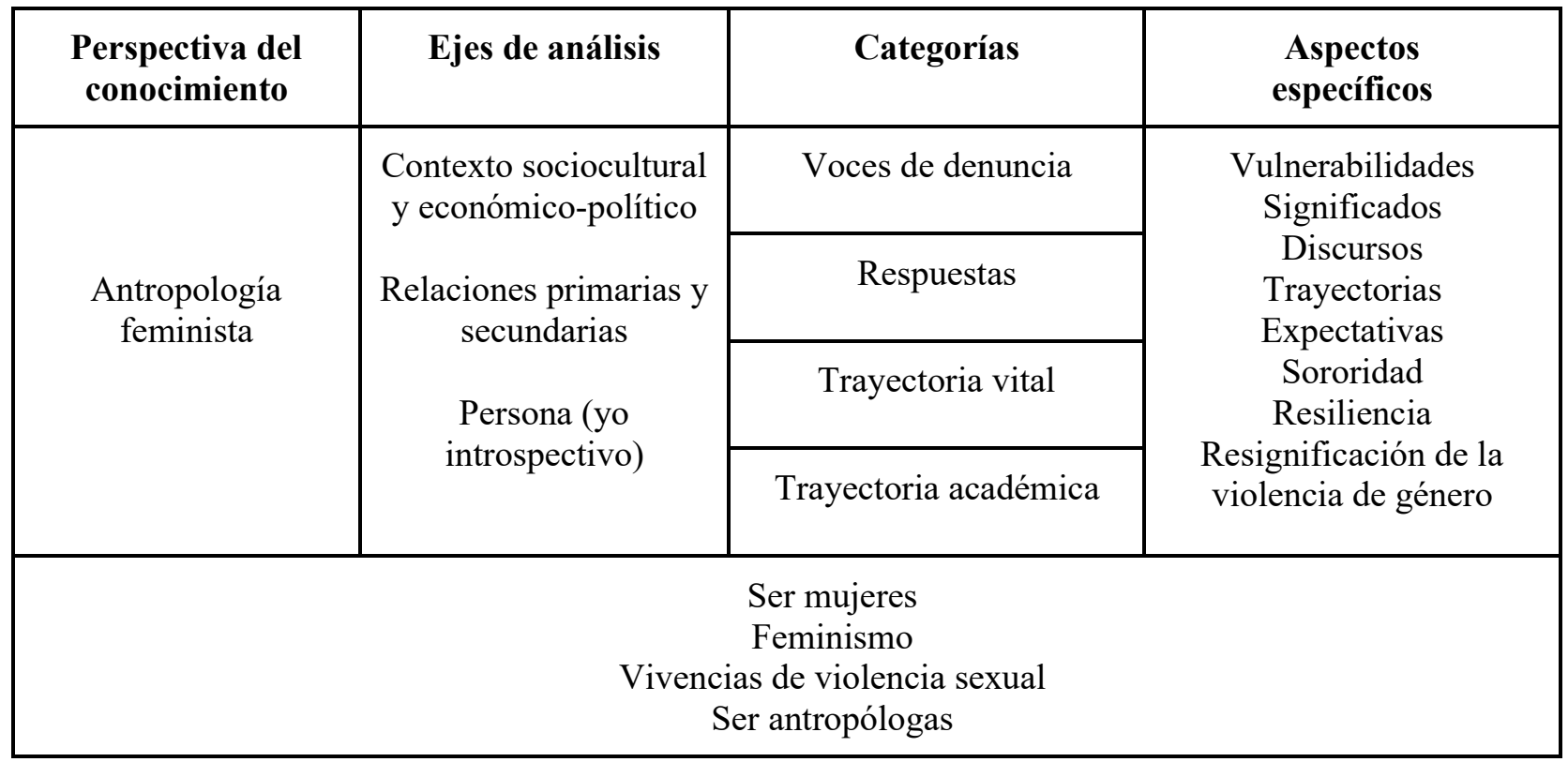

Fuente: elaboración propia 


\section{Resultados y discusión}

Después de haber invertido tiempo, esfuerzo y recursos en preparar un proyecto etnográfico, aunque en momentos distintos, ambas llegamos a una comunidad originaria en Perú para realizar trabajo de campo con ayuda de J, un antropólogo que estudiaba el folclore local para una agencia estatal. Algo común en ambas antropólogas, en ese momento, era la edad, la nacionalidad extranjera en Perú, la desconexión con nuestras redes sociales, académicas y familiares, ya que el pueblo estaba prácticamente incomunicado (sólo había un teléfono para uso comunal en el espacio público de una tienda) y la ilusión por realizar investigación en una zona poco explorada etnográficamente. Estos aspectos se convirtieron en vulnerabilidades en el contexto de campo.

A las dos, J nos presionó por sexo a cambio de poder permanecer en el trabajo de campo, nos degradó a objetos sexuales, intentó aislarnos dentro de la comunidad con intimidaciones e imposición de reglas y pretendió controlar nuestra producción académica.

Las escasas semanas que pasé allí [J] me hizo comentarios aludiendo a la tentación llegada del cielo que yo era para él. Seguía sin importar mi investigación; de hecho, nunca llegué a hacer una sola entrevista. Yo sólo era vista como un ser inferior y un objeto sexual para él.

La comunidad estaba en la sierra, por el día el sol era arrasador, pero en la noche las temperaturas bajaban mucho. El colchón de paja en el que descansaba no ayudaba mucho a guardar el calor. Cuando hacía referencia al frío que sentía, J me decía que si yo quería él podía dormir conmigo para darme calor. [...] Mi negativa le causaba gracia. [...] Todas las noches que pasé en esa cama de colchón de paja, ponía unas tijeritas debajo de la almohada como medida de defensa por si alguien entraba a agredirme. Ahora se ve como algo ridículo, ya que las tijeras eran tan pequeñas que no hubieran servido para nada, pero en ese momento era lo único que tenía a la mano para defenderme. 
A los pocos días de llegar, fuimos a otra comunidad (no recuerdo cómo se llamaba). Para llegar ahí caminamos todo el día por la sierra. No tengo presente la cantidad de horas que caminamos, pero sí el cansancio que sentía cuando llegamos a este pequeño pueblo. Las y los maestros de esta comunidad fueron muy amables, ofreciéndonos comida y donde pasar la noche. J se negó a que durmiéramos en casa de una maestra que, viendo el estado de agotamiento en el que llegué, enseguida abrió las puertas de su casa. Pasamos la noche en el laboratorio de la escuela de la comunidad. Al acostarme en el suelo sentí tanto miedo de estar sola con él en la misma habitación que me enfermé y pasé la noche en el baño.

[...] Al llegar una noche a las habitaciones, mientras estaba sacando algo de mi maleta, tocó la puerta y entró. Cerró (más bien, entornó la puerta, pues sin el tornillo no se podía encajar ${ }^{5}$ ) y se sentó en una cama que había al lado de la puerta, su intención era que nos organizáramos sobre las siguientes entrevistas que haríamos a las y los pobladores de la comunidad. Yo no me senté. Quizá mi instinto me mantuvo de pie y un paso atrás. Al levantarse, empezó a comentar que si no quería dormir con él en su habitación. Le dije que no, sin ser demasiado agresiva en mi respuesta. Entiendo que eso fue una estrategia para no enojarle. Pero mi intento fue fallido, ya que se abalanzó sobre mí para intentar besarme y sujetarme de los brazos. Yo giré la cara, me aparté y me fui hacia atrás, empujándole con todas mis fuerzas. Él se quedó quieto. Imagino que pensando qué hacer. Doy gracias al universo por lo que no sucedió después. Se fue molesto, el gesto le cambió y al salir de la habitación me dijo: "Puta". Esa noche no sólo puse el tornillo aquel viejo, sino que puse todo mi cuerpo contra la puerta, dormí sentada apoyada en ella.

\footnotetext{
${ }^{5}$ Las dos habitaciones que habíamos rentado estaban en la misma casa, pared con pared. Mi habitación tenía una puerta que daba directamente a la calle y que no tenía ningún cerrojo o algo para poder asegurar que la puerta no se abriese desde fuera. Busqué algo que poner a la puerta para no sentirme tan desprotegida $\mathrm{y}$, por suerte, encontré un tornillo viejo y alargado que me permitió hacer un agujero en la pared y otro en la puerta para encajarlo cuando estuviera dentro de la habitación y sentirme un poco más segura (Relato A).
} 
Tras dos o tres días [...] J me dijo que ya nunca más volvería, que hiciera las maletas y no regresara jamás. Me quedé en la ciudad más cercana. Aún faltaban más de dos meses para que saliera mi vuelo de regreso a España. Estuve unos días allí hasta que decidí ir a Lima. Me sentí muy mal, muy perdida, muy sola, gastando los recursos que había ahorrado (Relato A).

Cuando viajé a Perú para encontrar un lugar donde hacer mi investigación tuve un accidente en un autobús; más de veinte personas perdieron la vida. Después de un mes de recuperación en hospitales, decidí que continuaría con el trabajo de campo [...] En Lima, unas semanas después me encontré con $\mathrm{J}$, por consejo de mi director, quién me haría el favor de llevarme a la comunidad donde se encontraba realizando trabajo de campo.

Desde nuestro primer encuentro en Lima, J empezó a hacerme propuestas que yo decliné varias veces de forma cordial, apelando a su condición de hombre casado, las consideré normales como tantas otras insinuaciones no deseadas de hombres con los que había tenido que lidiar a lo largo de mi vida. Sin embargo, ya en la capital del distrito, J me dejó claro que el componente sexual era un requisito de acceso al campo, y yo entonces pensaba que no podía permitirme fracasar en mi empeño en tales circunstancias. Herida como estaba, con dos costillas rotas, múltiples hematomas y el trauma del accidente a cuestas, habiendo perdido mis cosas y mis ahorros, lo permití.

Durante este primer trabajo de campo, vi a J en tres ocasiones más en las que me utilizó para ser su camarógrafa, para cargar su grabadora y asistirlo en su investigación. La mía, sobre el embarazo y el parto, poco avanzó y él siempre estaba presente en las entrevistas. Yo fui además a hacer investigación de archivo y trabajo de campo en otro núcleo importante de la sierra, hasta completar tres meses de trabajo. Hubo muchos intentos de control sobre mí y mi investigación que entonces yo no supe ver pero que fueron evidentes cuando yo intenté terminar el affaire. 
Después de tres meses, en los que logré un puñado de entrevistas con parteras donde hice trabajo de campo sola, regresé con mi familia, y posteriormente a mi universidad en España. Aunque la relación continuó por un tiempo a distancia, con la perspectiva del paso de los días comprendí que J se había aprovechado de mí y corté la relación. A pesar de sus resistencias iniciales, logré que disminuyeran las comunicaciones y llegué a pensar que las cosas habían acabado para bien. Un año después, una vez que sustenté mi tesina, contacté de nuevo con J para comentarle sobre mis planes de regreso para proseguir mis estudios de doctorado. Unos días antes de mi viaje a Perú [...] J me llamó para prevenirme por si venía alguien a hablarme de una mujer "loca" (refiriéndose a A) que decía que él la había sacado del trabajo de campo; y, por supuesto, para pedirme que hablara en su favor. El caso vine a conocerlo más ampliamente en Lima por mi director de tesis, quien me preguntó si J me había maltratado o humillado, sin hacer ninguna mención al acoso sexual. Le dije que no, aunque sabía que [...] sí lo había hecho, porque también lo había hecho conmigo. Le dije que no porque no quería verme expuesta y pensé que con esto $\mathrm{J}$ definitivamente me dejaría en paz y saldría de mi vida. Pero no fue así.

En Lima también hablé con J y le pedí que por favor me dejara en paz, aunque siguió escribiéndome, llamándome y yendo a buscarme, incluso cuando ya no era antropólogo de campo (Relato B).

De formas distintas ambas nos negamos a esta presión y sufrimos violencia psicológica y física; incluso, para B, también una serie de retaliaciones e intimidaciones hasta la actualidad. Aunque el contexto era diferente al del marco jerárquico institucional de la universidad, J tenía "poder" sobre nosotras porque no conocíamos la zona. Durante la estancia en el campo vivimos una situación de vulnerabilidad y de dependencia en la búsqueda de relaciones horizontales e impredictibilidad constante (Schneider, 2020). Se desplegó un control masculino hacia lo femenino en distintos ámbitos: el del cuerpo, el del territorio, el de la jerarquía "institucional" y el del reconocimiento profesional. 
Ante mi resistencia y mi creciente independencia, empezó a hacerme exigencias fuera de toda razón sobre mi labor y mis actividades, me amenazó con sacarme del trabajo de campo. Llegó a pedirle a personas que no me invitaran a actividades donde yo podría bailar con hombres y me amenazó al menos en dos ocasiones con matarme si llegaba a saber que estaba con otro hombre. Me exigía informarle sobre mis actividades académicas y movimientos, y me insultaba si no lo hacía. [...] le rogué e insistí que por favor me dejara en paz. Pero no lo hizo, regresó al campo. Me obligó a mantener relaciones sexuales con él, aunque yo me negué, entonces me asfixió hasta que perdí el conocimiento y me recordó que me mataría si me veía con otros hombres. La situación llegó a un momento crítico en su segunda visita, durante un ataque de ira y celos, porque había perdido el control sobre mí, J me sacó de la casa de la enfermera del pueblo, y me llevó — casi arrastró - hasta mi casa. Allí me insultó y empujó, dañó varios objetos y amenazó con destruirme, acabar con mi trabajo, con sacarme del campo y, nuevamente, con matarme. [...] El nivel de violencia fue tal que yo entré en una especie de ataque de pánico y ansiedad (Relato B).

Al llegar a la comunidad las reglas impuestas por $\mathrm{J}$ empezaron a surgir, por ejemplo, no podía salir a caminar por el pueblo sola, no podía hablar con las personas de allí si él no estaba presente, trabajaría mi tema después de que se hubieran hecho las entrevistas que nutrirían su trabajo, debía tener mucho cuidado de acercarme y hablar con la policía porque mis formas eran coquetas... Como éstas muchas otras advertencias o amenazas que con dulces palabras, y siempre por mi bienestar y seguridad, yo acaté sin rechistar (Relato A). 


\section{Nuestras voces de denuncia y sus silencios}

Cada ejercicio de poder y violencia contra nosotras fue distinto, a pesar de enmarcarse en un mismo contexto; hay una diferencia en la manera en que tratamos la denuncia y en cómo nos respondieron las personas responsables y las que conocieron nuestras experiencias. Nuestras voces de denuncia, acompañadas de sentires, nacen en dos espacios: en Perú y en nuestra universidad madrileña. Narraremos a continuación lo ocurrido en Perú:

En Lima, escribí un mail a la persona que me había contactado con J y su respuesta fue remitirme a un profesor que trabajaba en un centro de investigación. El contacto sirvió para contar una vez mi historia y ya.

De casualidad venía el director del Departamento donde estudiaba mi posgrado para participar en un evento académico y decidí hablar con él. Tras su ponencia, durante el descanso, me acerqué y le comenté a grandes rasgos. Su respuesta fue que tenía que haberle empujado. Eso fue todo. Nunca más me preguntó nada, no se interesó por mi estado o por si podía apoyarme con algo; mucho menos, puso en tela de juicio el acoso de J, sino todo lo contrario, puso en tela de juicio mi respuesta, quitándole importancia a lo sucedido. Para colmo, una estudiante de doctorado de la misma universidad, a quien me encontré en un supermercado, [y a quien le habían contado de mi caso] cuestionó lo extraño de lo que me había pasado, porque J era una persona educada y respetuosa. Creo que me sentí mucho peor aún de lo que ya estaba. Esos comentarios no sólo defendían a un acosador [...], sino que me dejaban a mí abiertamente como mentirosa (Relato A).

Yo estaba aterrorizada. En ese momento decidí, por mi integridad personal y para proteger mi trabajo, comentárselo a mi director de tesis, [por medio de] una carta [donde] se omitía la parte del affaire con este hombre en mi primer trabajo de campo e incluso el acoso sexual en su conjunto, ya que 
no quería verme expuesta públicamente, tenía miedo y vergüenza. Estaba en un país extraño y [...] me había amenazado de muerte.

Escribí tres correos electrónicos y le hice una llamada telefónica antes de la carta "oficial" diciéndole que la situación era muy complicada, por las amenazas de $\mathrm{J}$ de sacarme del campo y por las exigencias acerca de mi trabajo. Él hizo caso omiso, normalizándolo y finalmente diciendo que era un asunto personal. Por supuesto, ¡cómo me iba a ayudar un hombre que había tratado de besarme en los elevadores del sótano de la facultad! Gracias a la denuncia de una persona que también trabajaba para la misma institución que $\mathrm{J}$ y que fue testigo parcial de los hechos, J fue investigado y yo di mi testimonio. Por supuesto, fui nuevamente amenazada por él para que me retractara, pero no lo hice. Finalmente, el acoso fue comprobado y J fue "invitado a renunciar" de su cargo (Relato B).

\section{En Madrid}

Fui a exponer lo sucedido por dos vías diferentes. La primera persona a quien acudí para informar, ni siquiera para solicitar nada ni poner denuncia, fue al académico que me había contactado con $\mathrm{J}$ meses atrás. Tras narrar mi vivencia lo único que recibí fue un pañuelo para secarme las lágrimas y una petición de pruebas sobre lo ocurrido. Mi palabra no valía nada. La de J superaba con creces la mía.

Tras esta negativa fui a mi universidad (aún la considero mi universidad, a pesar del poco apoyo que obtuve) a poner una denuncia formal ante la oficina de atención a estudiantes, a donde me canalizó una profesora del mismo departamento, quien además de animarme a que esto no quedara impune, dijo que, si $\mathrm{J}$ se atrevía a volver a la facultad, ella misma se encargaría de poner carteles con su foto para que todo el mundo supiera lo que había hecho. 
Antes de contar nuevamente mi experiencia en el trabajo de campo, la mujer que me atendió en esta oficina me preguntó si el acosador era mi director de tesis, quien ya había tenido otras denuncias. Yo me quedé impactada, ya que me llevaba bastante bien con él. Había sido mi profesor en el posgrado y yo había decidido que fuera el director de mi tesis. Me sorprendió mucho y lo negué. Dije que el acosador había sido J. La respuesta fue que la denuncia no podía tener trámite, ya que él no tenía vinculación con la universidad en ese momento.

No me quedé tranquila, pero creí que había hecho todo lo posible. A los pocos días quedé con mi director de tesis, a quien le conté otra vez todo. Su respuesta me dejó helada. Dio a entender que no podía esperar otra cosa, que él también estaba esperando que yo le diera un "beso en condiciones" cada vez que me despedía de él después de las asesorías. [...] Me fui a casa realmente hecha una mierda (Relato A).

En la experiencia de A, la ayuda provino de la familia y amistades en un espacio temporal posterior a la situación de acoso (en Madrid). En Lima cabe destacar, a propósito de la cercanía territorial, la figura de una persona del equipo de trabajo del victimario, quien fue testigo de algún episodio de violencia y su amabilidad fue un punto de apoyo importante.

En el caso de B, sí se vivió la solidaridad de las mujeres de la comunidad en Perú, quienes conocieron la historia, la reconfortaron y la protegieron de J. Para las mujeres de esta zona, la violencia sexual es frecuente; la violación es una forma de conseguir matrimonio y la violencia intrafamiliar es tristemente común. A diferencia de nuestra sociedad y en lo que nosotras mismas experimentamos, en ese contexto de la comunidad originaria nunca es puesto en duda el testimonio de la víctima.

Tras la denuncia, la primera reacción de $\mathrm{J}$ fue tildarnos a las dos de locas y mentirosas. Aunque había dos testimonios en contra de su actuar, los tutores responsables de nuestra formación universitaria normalizaron los hechos. 
En ambos casos las respuestas de la universidad fueron negativas; existió una minimización de la gravedad de las experiencias que vivimos, siendo ésta la defensa del acosador, sustentando su inocencia en la falta de pruebas y en la posición académica que construía o mantenía el victimario. Además, se defendió la idea del derecho de los hombres a comportarse como quieran y la obligación de las mujeres a convenir cuando cesa ese comportamiento, recayendo la responsabilidad final en ellas, sin juzgar el hecho o actitud masculina. Sólo destacó la respuesta de la institución pública peruana, donde fue forzado a renunciar a su cargo.

En ambos casos destaca el mismo discurso que ampara las respuestas negativas. Esto apoya la idea del continuum (Kelly, 1988). A pesar de multisituarnos en distintos espacios (Perú y Madrid) las respuestas y el apoyo a la agresión fueron tejidas bajo un mismo patrón de violencia estructural, incluso llegando a tener "recompensa social" y "satisfacción personal" a pesar de que este tipo de comportamientos están teóricamente rechazados (Juliano, 2011, p. 24). Aunque $J$ fue despedido de la institución en la que trabajaba, en el ámbito académico su accionar no ha tenido impacto y ha seguido vinculado a distintas instituciones.

El cuidado que ambas mantuvimos en los términos, en el proceso de denuncia y en la vía de expresar nuestro dolor, no se refleja de vuelta por parte de las instituciones ni de los investigadores involucrados; contrariamente a la privacidad que habíamos otorgado (y que seguimos incorporando), el resto de los actores no tuvieron el respeto que merecíamos y contaron, con nombres y apellidos, nuestras experiencias a personas ajenas.

\section{Trayectorias: resiliencia y resignificaciones}

Las consecuencias de la violencia sexual son diversas, aunque destacan las secuelas psicológicas, los trastornos emocionales, la tristeza, la depresión, la ansiedad, el miedo, la baja autoestima, entre otras (Igareda y Bodelón, 2013), afectando no sólo al rendimiento escolar y profesional, sino también a las relaciones sociales y familiares. En nuestros casos los efectos de los episodios de violencia no recayeron sólo en la pérdida del rumbo personal, 
sino también en nuestra trayectoria profesional. Se tambalearon las metas trazadas anteriormente y las expectativas vitales se desdibujaron.

Después de todo, por mi cabeza pasaban muchas cosas. Había momentos en los que no sabía cómo articular estos acontecimientos. [...] Decidí no volver a Perú y abandonar mi tema de investigación, algo que adoraba (Relato A).

La falta de apoyo de mi director significó que yo solicitara primero su cambio, luego una cotutela con otra universidad donde continué mis estudios de doctorado. J me empezó a acosar en los congresos a los que asistía, con cosas como ofrecerse como moderador en la mesa en la que yo participaba, persiguiéndome cuando iba a los servicios, hasta que desistí de acudir a grandes eventos. Además, se ha dedicado a utilizar espacios académicos como forma de hacer ataques ad hóminem (mejor dicho, ad mulierem) contra mí. Las represalias de $\mathrm{J}$ han tenido un profundo impacto en mi vida y en mi carrera (Relato B).

Tras los sucesos, en el caso de A, hubo un cese en la consecución de metas académicas y una ruptura tajante con Perú. Durante varios meses las expectativas se replantearon ante la pérdida de rumbo profesional. La asociación simbólica de Perú con lo vivenciado en el trabajo de campo era innegable y, aunque había otras posibilidades de continuar estudiando la temática, el desánimo opacó toda ilusión de seguir. La carrera académica continuó en México.

Para B, el acoso continúa hasta la fecha en que escribimos este artículo, ya que ella no renunció al trabajo de campo. La carrera académica quedó en paréntesis por la presión sentida, dibujándose otras opciones profesionales en el horizonte.

Las trayectorias vitales de los dos casos muestran una consonancia en las consecuencias de la violencia sexual desarrollada en una primera etapa: la impotencia, el insomnio, el estrés, 
la tristeza y el miedo, afectando nuestra capacidad emocional y física, aunque en diferentes tiempos y bajo circunstancias contextuales únicas. La no comprensión total de los acontecimientos, siendo protagonistas de éstos, nos posicionó en una búsqueda constante de respuestas, obteniendo un rechazo tras otro. Esto minó nuestra autoestima como mujeres al identificar la desvalorización que el resto de los actores sociales nos otorgaban y la humillación hacia nuestras personas. "Así me sentía: culpable, avergonzada, sin entender muy bien nada, tonta por no haberme dado cuenta antes, sucia por provocar este tipo de comportamientos en los hombres, vulnerable y pequeña, muy pequeña” (Relato A).

El corte del discurso machista que estaba detrás de las respuestas nos posicionaba, sutilmente, en ser las culpables de la violencia: por no haber sabido actuar, por haber provocado esa situación y por no haber sabido decir "no" a tiempo. Esta revictimización hizo eco en el sentimiento de vergüenza. "Denunciar violencia sexual es una ordalía para las sobrevivientes. Somos acusadas de mentirosas, tachadas de locas y exageradas, y culpabilizadas por lo que nos sucede" (Relato B).

La autoculpabilización, con grados diferenciados para cada una de nosotras, provino del discurso social apoyado en los comportamientos externos (Rodríguez, 2015), en la imagen de "locas, frágiles, malas, exageradas y mentirosas" detrás de las respuestas y en la vergüenza sentida desde nuestros cuerpos y nuestras mentes por lidiar con el papel de víctimas.

Me hago la misma pregunta que Eva Moreno en el relato del acoso y la final violación que sufrió durante su trabajo de campo en Etiopía: ¿Tal vez yo debí reaccionar antes o de otra manera? Sin duda debí ser más valiente y hablar antes, pero no pude. Me tardé en reconocer mi situación y tuve pánico a ser culpabilizada (Relato B).

Ahora sé que fue una estrategia de supervivencia para no tener que recordar ni hacer frente a ningún otro comentario que me pusiera en duda. Cerré esa etapa, quizá no de la mejor manera, de la forma en que pude. Me alejé de todo y no lo enfrenté (Relato $\mathrm{A}$ ). 
Por medio de lecturas, charlas, autoexploración personal y social se alcanza una más clara identificación del conflicto, del posicionamiento pasado y presente y de las vulnerabilidades. El rescate de las emociones sentidas en los diferentes niveles de la vivencia (física, emocional, estructural y simbólica) y la autocrítica se conforman como las vías para el acercamiento a la construcción de sentido de nuestros planteamientos personales, nuestras relaciones, nuestra aceptación y nuestros retos, creando espacios de reflexión significativos para la sanación de un evento traumático (Agudelo y Estrada, 2013).

La plataforma digital \#MeToo ha sido también una vía para resignificar nuestras experiencias y lidiar con los episodios de violencia sexual. A partir de un encuentro sororo, nuestras vivencias se transformaron en "generalidad", desdibujándose el sentir de "unicidad", rompiendo la "individualización del fenómeno" como algo aislado y vergonzoso, se insertaron otros sentidos que destruyeron la culpa a partir de identificar el panorama amplio de la dominación masculina como algo estructural (macro) y sólo personal (micro) desde la acción puntual. En este sentido, un cúmulo de acciones, actitudes, ejercicios y construcción de relaciones se dieron cabida en un espacio temporal y contextual, sentido de manera individual, pero expresado y compartido con una globalidad conectada, para avanzar en la superación de la violencia.

En este proceso se resignifica el suceso, el sentir y el actuar, posicionándonos ante la lucha feminista, porque lo personal es político (lema de la segunda ola feminista) y social. Queremos denunciar, ser escuchadas y participar en el debate de la desigualdad de género como una urgencia social. Las dos antropólogas nos construimos a partir del acontecimientoverdad como sujetas reflexivas y sujetas con sentido (Zizek, 1999) desde la sororidad, donde convergen el compañerismo, la solidaridad y la empatía, tanto en la calma como en la ira.

Me alegro de estar casi diez años después sentada frente a esta página en blanco de mi historia porque ahora tengo más experiencia, más fuerza, menos vergüenza, menos lágrimas y soy más sorora. Éste no es sólo un relato de denuncia, sino también de lucha, de reflexión individual, de crítica social y de solidaridad entre mujeres (Relato A). 
Enfrentar el rechazo y la ignorancia hacia nuestras voces generó resiliencia y cada una tomó rumbos diferentes para sanar y superar desde un proceso de no culpabilización. Actualmente, nos seguimos resignificando y auto-socio-posicionando como mujeres denunciantesanalíticas capaces de transformar ese "sin-sentido-dolor-vergüenza" en una apropiación propositiva en el campo de las relaciones igualitarias entre mujeres y hombres.

\section{Conclusiones}

Nuestros motivos para escribir este relato resuenan con los de Bjerén (2017), quien destaca dos razones para haber escrito Rape in the Field (1995): la venganza, queriendo avergonzar a su violador, y la necesidad de advertir a las jóvenes investigadoras sobre los peligros del estereotipo del etnógrafo como un "lobo solitario" imbuido en la ficción del género neutral.

Esta reflexión no sólo apunta al acosador, sino a todas las personas que corrieron un tupido velo ante estos casos minusvalorando nuestras voces (como mujeres estudiantes), dando cuenta una vez más de la jerarquía androcéntrica existente en la universidad y de la complicidad entre algunos hombres en la academia. El nulo cuestionamiento sobre la actitud y acciones del victimario deja entrever la alianza masculina (del no hacer nada) y niega un juicio sobre su ética por su posicionamiento como investigador.

Respecto al papel que juegan las instituciones de educación superior en España, y también en Latinoamérica, no hay una certeza de que los centros que impulsan y promueven el conocimiento tengan lineamientos claros para asegurar la ética de los investigadores acusados de violencia. Se sigue sustentando un discurso, promovido por el ámbito jurídico, basado en la denuncia con pruebas y testigos. Esto no sólo revictimiza a la mujer acosada, sino que en las comunidades donde se realizan trabajos de campo es complejo tener este tipo de demostraciones "objetivas".

De acuerdo con esta visión, compartimos una propuesta con algunos puntos que contribuirían al cambio en el devenir de la antropología: 
a) Revisar la forma en que se enseñan los textos clásicos de antropología con la finalidad de analizar críticamente el androcentrismo blanco inmerso en los mismos, debido a la urgencia de cuestionar las raíces epistemológicas coloniales de la disciplina y de incorporar la perspectiva de género en la teoría y en la práctica del trabajo de campo.

b) Brindar capacitación continua sobre temas de género y violencia a la planta docente, administrativa y de servicios de los centros universitarios; particularmente a aquellos/as investigadores/as que colaboren con estudiantes de instituciones externas en el trabajo de campo.

c) Las facultades o departamentos de antropología deben crear un registro de la planta académica externa con quien la población estudiante y docente colabora académicamente para realizar trabajo de campo. Las redes de comunicación y apoyo deben ser constantes con investigadores/as e instituciones.

d) Crear una oficina de atención en el departamento de antropología, en el campus o en la universidad, dirigida y atendida por personas capacitadas en el tema de violencia de género, donde se garantice la confidencialidad y el acompañamiento. Asimismo, sería conveniente dar difusión de este servicio y de los derechos, respaldados a su vez por un protocolo de atención ante episodios de violencia hacia las mujeres.

e) Proponer diálogos disciplinares y políticos que aborden y reconozcan casos específicos de violencia sexual durante los estudios universitarios y en el trabajo de campo.

Desde nuestro rol de investigadoras y como protagonistas del fenómeno, hemos mantenido una conversación dialógica donde los significados de la violencia y las representaciones sociales de género se han reformulado (Pérez, Rábago y Castillo, 2020). A partir de los conflictos surgidos, nos hemos conformado como ciudadanas autónomas y con legitimidad dentro de los contextos de política (Vázquez, 2006) y este esfuerzo autoetnográfico también pretende coadyuvar a "crear las condiciones para una conciencia crítica capaz de imaginar una política radical de las posibilidades" (Denzin, 2017, p. 87). En tenor de esto, y tomando el continuum de la violencia sexual como un problema sociopolítico, participamos en la necesidad de compartir las situaciones vividas por muchas mujeres antropólogas en el campo durante su carrera formativa y profesional con el fin de contribuir a una reflexión en nuestra 
disciplina sobre cómo se piensa y se enseña el trabajo de campo. Destacan, asimismo, la autoetnografía y la sororidad como herramientas metodológicas en la construcción de conocimiento antropológico y por su potencial transformador (Poó, 2009).

Este artículo se construye a partir del encuentro sororo entre las dos autoras después de una década de distanciamiento, del reconocimiento de dos historias de violencia y de la necesidad de la solidaridad para la transformación de nuestra disciplina. Desde nuestras vivencias, nos sumamos a la crítica feminista y decolonial para comprender el problema de la violencia de género en el campus y en el campo y a la propuesta de diálogo para deconstruir y replantear la forma en que pensamos.

\section{Referencias bibliográficas}

Agudelo, María Eugenia y Estrada, Piedad. (2013). Terapias narrativa y colaborativa: una mirada con el lente del construccionismo social. Trabajo Social, 29(29), 15-48. Recuperado de https://revistas.upb.edu.co/index.php/trabajosocial/article/view/2437

Arfuch, Leonor. (2012). Narrativas del yo y memorias traumáticas. Tempo e Argumento. Revista do Programa de Pós- Graduação em História, 4(1), 45-60. doi: $10.5965 / 2175180304012012045$

Berry, Maya; Chávez, Claudia; Cordis, Shanya; Ihmoud, Sarah y Velásquez, Elizabeth. (2017). Toward a Fugitive Anthropology: Gender, Race, and Violence in the Field. Cultural Anthropology, 32(4) 537-565. doi: https://doi.org/10.14506/ca32.4.05

Bjerén, Gunilla. (2017). Comments on "Rape in the field. Reflections from a survivor". Cadernos de Campo, 26(1), 265-269. Recuperado de https://www.revistas.usp.br/cadernosdecampo/article/view/143216

Blanco, Mercedes. (2012). ¿Autobiografía o autoetnografía? Desacatos, 38, 169-178. doi: https://doi.org/10.29340/38.278 
Blanco, Mercedes. (2012b). Autoetnografía: una forma narrativa de generación de conocimientos. Andamios, Revista de Investigación Social, 9(19), 49-74. doi: http://dx.doi.org/10.29092/uacm.v9i19.390

Bénard, Silvia. (2019). Autoetnografía. Una metodología cualitativa. México: Universidad Autónoma de Aguascalientes y El Colegio de San Luis.

Camacho, Hermelinda y Marcano, Noraida. (2003). El enfoque de investigación introspectiva vivencial y sus secuencias operativas. Algunos casos de estudio. Omnia, 9(1), 1-22. Recuperado de https://www.redalyc.org/articulo.oa?id=73711580002

Chang, Heewon. (2008). Autoethnography as method. Walmut Creek, CA: Left Coast Press.

Delory-Momberger, Christine y Betancourt, Miguel Orlando. (2015). El relato de sí como hecho antropológico. En Gabriel Jaime Murillo (Comp.), Narrativas de experiencia en educación y pedagogía de la memoria (pp. 57-68). Buenos Aires: Universidad de Buenos Aires y Consejo Latinoamericano de Ciencias Sociales y Universidad de Antioquia. Recuperado de https://docplayer.es/54187041-2-el-relato-de-si-comohecho-antropologico.html

Denzin, Norman. (2017). Autoetnografía Interpretativa. Investigación Cualitativa, 2(1), 81-90.

Douglas, Kitrina y Carless, David. (2013). A History of Autoethnographic Inquiry. En Stacy Holman Jones, Tony E. Adams y Carolyn Ellis (Coords.), Handbook of Autoethnography (pp. 84-106). London: Routledge. Recuperado de https://www.routledgehandbooks.com/doi/10.4324/9781315427812.ch2

Dubé, Gabrielle. (2017). La auto-etnografía, un método de investigación inclusivo. Visión docente Con-Ciencia, XV(83), 6-23. Recuperado de https://docplayer.es/83787575La-auto-etnografia-un-metodo-de-investigacion-inclusivo-dra-gabrielle-dube.html 
Ellis, Carolyn. (2009). Telling tales on neighbors: Ethics in two voices. International Review of Qualitative Research, 2(1), 3-27. doi: https://doi.org/10.1525/irqr.2009.2.1.3

Ellis, Carolyn; Adams, Tony y Bochner, Arthur (2015). Autoetnografía: un panorama. Astrolabio, $\quad 14, \quad 249-273 . \quad$ Recuperado de https://revistas.unc.edu.ar/index.php/astrolabio/article/view/11626/12041

Escobar, Natalia. (2018). ¡No Es Mi Culpa! Enfrentando el acoso sexual y la violencia de género en el trabajo de campo. Cadernos de campo, São Paulo, 27(1), 256-273. doi: 10.11606/issn.2316-9133.v27i1p256-273

Espitia, Ingrid; Ojeda, Diana y Rivera, Claudia. (2019). La "princesa antropóloga": disciplinamiento de cuerpos feminizados y método etnográfico. Nómadas, 51, 99115. doi: 10.30578/nomadas.n51a6

Esteban, Mari Luz. (2004). Antropología encarnada. Antropología desde una misma. Papeles del $\quad$ CEIC, $12,-21 . \quad$ Recuperado de https://ojs.ehu.eus/index.php/papelesCEIC/article/viewFile/12093/11015

Fileborn, Bianca y Loney-Howes, Rachel. (2019). \#MeToo and the Politics of Social Change (eBook). Suiza: Palgrave Macmillan. Recuperado de https://doi.org/10.1007/978-3$\underline{030-15213-0}$

Finlay, Linda. (2002). Negotiating the swamp: the opportunity and challenge of reflexivity in research practice. Qualitative Research, 2(2), 209-230. doi: https://doi.org/10.1177/146879410200200205

Grant, Alec. (2010). Autoethnographic ethics and rewriting the fragmented self. Journal of Psychiatric and mental health nursing, 17(2), 111-116. doi: 10.1111/j.13652850.2009.01478.x 
Gregorio, Carmen. (2014). Traspasando las fronteras dentro-fuera: Reflexiones desde una etnografía feminista. AIBR, Revista de Antropología Iberoamericana, 9(3), 297-322. doi: 10.11156/aibr.090305

Guerrero, Joaquín. (2014). El valor de la auto-etnografía como fuente para la investigación social: del método a la narrativa. Azarbe, Revista internacional de Trabajo Social y Bienestar, 3, 237-242. Recuperado de https://revistas.um.es/azarbe/article/view/198691

Huang, Mingwei; Lu, Vivian; Macdougall, Susan y Steffen, Megan. (2018). Disciplinary Violence. Anthropology News, 59(3), 79-82. doi: https://doi.org/10.1111/AN.861

Igareda, Noelia y Bodelón, Encarna. (2013). Las violencias sexuales y el acoso sexual en el ámbito universitario español. Rivista di Criminologia, Vittimologia e Sicurezza, VII(2), 65-72. Recuperado de https://core.ac.uk/download/pdf/33153115.pdf

Juliano, Dolores. (2011). Cultura y sexualidad. En Cristina Villalba y Nacho Álvarez (Coords.), Cuerpos políticos y agencia. Reflexiones feministas sobre cuerpo, trabajo y colonialidad (pp. 21-42). Granada: Universidad de Granada.

Kelly, Liz. (1988). Surviving Sexual Violence. Minnesota: University of Minnesota Press.

Kloß, Sinah Theres. (2017). Sexual(ized) harassment and ethnographic fieldwork: a silenced aspect of social research. Ethnography, 18(3), 396-414. doi: https://doi.org/10.1177/1466138116641958

de Lauretis, Teresa. (1986). Feminist Studies/Critical Studies: Issues, Terms, and Contexts. En Teresa de Lauretis (Ed.), Feminist Studies/Critical Studies (pp. 1-19). London: Palgrave Macmillan UK. Recuperado de https://link.springer.com/chapter/10.1007/978-1-349-18997-7_1 
Lonergan, Bernard. (2001). Método en Teología. Salamanca: Ediciones Sígueme.

Mendes, Kaitlynn; Ringrose, Jessica y Keller, Jessalynn. (2018). \#MeToo and the promise and pitfalls of challenging rape culture through digital feminist activism. European Journal of Women's Studies, 25(2), 236-246. doi: https://doi.org/10.1177/1350506818765318

Moreno, Eva. (2005). Rape in the field. Reflections for a survivor. En Don Kulick y Margaret Willson (Eds.), Taboo: Sex, identity and erotic subjectivity in anthropological fieldwork (pp. 219-250). London: Routledge.

Mulinari, Diana y Sandell, Kerstin. (1999). Exploring the Notion of Experience in Feminist Thought. Acta Sociológica, 42(4), 287-297. Recuperado de https://www.jstor.org/stable/4201163

Nelson, Robin G.; Rutherford, Julienne N.; Hinde, Katie and Clancy, Kathryn B. H. (2017). Signaling Safety: Characterizing Fieldwork Experiences and Their Implications for Career Trajectories. American Anthropologist, 119(4), 710-722. doi: https://doi.org/10.1111/aman.12929

Pérez, Lucía; Rábago, Marcela y Castillo, Georgina. (2020). Equipo Reflexivo: Voces que Rescatan la Identidad Preferida de las Mujeres Universitarias. Multidisciplinary Journal of Gender Studies, 9(1), 51-78. doi:10.17583/generos.2020.4295

Poó, Candela. (2009). Qué puede un cuerpo (impaciente). Reflexiones autoetnográficas sobre el cuerpo y la enfermedad. Athenea Digital, 15, 149-168. doi: https://doi.org/10.5565/rev/athenead/v0n15.635

Rodríguez, Ricardo. (2015). Culpa, miedo y vergüenza. Las emociones de la violencia (El caso de violencia contra la pareja y/o ex -pareja). Derechos y libertades, 33, 223-252. doi: http://dx.doi.org/10.14679/1017 
Roscoe, Karen y Madoc, Iolo. (2009). Critical social work practice a narrative approach. International Journal of Narrative Practice, 1(1), 9-18. Recuperado de https://glyndwr.repository.guildhe.ac.uk/id/eprint/119/1/fulltext.pdf

Salter, Michael. (2019) Online Justice in the Circuit of Capital: \#MeToo, Marketization and the Deformation of Sexual Ethics. En Bianca Fileborn y Rachel Loney-Howes (Eds.), \#MeToo and the Politics of Social Change (pp. 317-334). Suiza: Palgrave Macmillan. Recuperado de https://doi.org/10.1007/978-3-030-15213-0

Schmerler, Gil y Steffen, Megan. (2018). The Disavowal of Henrietta Schmerler. Anthropology News, 59(3), 17-19. doi: https://doi.org/10.1111/AN.860

Schneider, Luisa T. (2020). Sexual violence during research: How the unpredictability of fieldwork and the right to risk collide with academic bureaucracy and expectations. Critique of Anthropology, 40(2), 173-193. doi: https://doi.org/10.1177/0308275X20917272

Scott, Joan W. (1991). The Evidence of Experience. Critical Inquiry, 17(4), 773-797. Recuperado de http://www.jstor.org/stable/1343743

Segato, Rita. (2010). Las estructuras elementales de la violencia. Buenos Aires: Prometeo.

Steffen, Megan. (Noviembre 13 del 2017). Doing Fieldwork After Henrietta Schmerler. On Sexual Violence and Blame in Anthropology. Recuperado de: https://americanethnologist.org/features/reflections/doing-fieldwork-after-henriettaschmerler

Tilley-Lubbs, Gresilda. (2015). La autoetnografía crítica y el self vulnerables como investigadora. REMIE, Multidisciplinary Journal of Educational Research, 4(3), 268285. doi: http://dx.doi.org/10.4471/remie.2014.14 
Vázquez, Francisco. (2006). El problema de la reflexividad en Pierre Bourdieu de la epistemología a la ética. Opinión jurídica, 5(10), 87-101.

Zizek, Slavoj. (1999). El sujeto espinoso: el centro ausente de la ontología política. Barcelona: Paidós.

\section{VIRGINIA ROMERO PLANA}

Es trabajadora social, antropóloga y doctora en Estudios mexicanos (Centro de Estudios Superiores e Investigación de Colima, 2015). Tiene un posgrado Especialista en Derechos Humanos: pasado, presente y futuro por la Universidad Nacional de Educación a Distancia de España (2009) y un posgrado Experto en Proyectos de Cooperación al desarrollo por la Universidad de Alcalá de Henares (2010). Sus líneas de investigación son la antropología de la pobreza, el género y la migración. Actualmente es profesora investigadora de tiempo completo en el departamento de Trabajo Social de la Universidad de Sonora. Dos de sus últimas publicaciones son: (2020). Violencia simbólica hacia las mujeres: un estudio de los comerciales de cerveza Tecate en México. Revista Prisma social, 30, 229-249. https://revistaprismasocial.es/article/view/3704/4358 (2019). Vivir bajo el cielo. Pobreza y masculinidad en las fronteras. México: Universidad de Sonora y Editorial Fontamara.

\section{LUZ MARTÍNEZ SANTAMARÍA}

Es licenciada en Historia, con especialidad en Antropología de América, y doctora en Antropología Social y Estudios Amerindios por la Universidad de St. Andrews (2020). Actualmente se desempeña como investigadora asociada del Museo Nacional de Sicán

(Perú). Sus áreas de interés son: antropología y etnohistoria andinas, antropología de la vida, decolonialidad, género, quechua. Dos de sus últimas publicaciones son: (2017). Música y cantos tradicionales de Incahuasi. Lima: Ministerio de Cultura de Perú. (2015). La Música el taki y la danza en Cañaris. En Ministerio de Cultura de Perú, Música y cantos tradicionales de Cañaris (pp. 25-41). Lima: Ministerio de Cultura de Perú. 